Open Access

\title{
Prognostic value of calcium score and coronary flow velocity reserve in asymptomatic diabetic patients
}

Miodrag Dikic ${ }^{*}$, Milorad Tesic $^{1}$, Zeljko Markovic ${ }^{2,3}$, Vojislav Giga ${ }^{1,2}$, Ana Djordjevic-Dikic ${ }^{1,2}$, Jelena Stepanovic ${ }^{1,2}$, Branko Beleslin ${ }^{1,2}$, Ivana Jovanovic ${ }^{1}$, Ana Mladenovic ${ }^{3}$, Jelena Seferovic ${ }^{2,4}$, Miodrag Ostojic ${ }^{2}$

and Aleksandra Arandjelovic ${ }^{2,5}$

\begin{abstract}
Background: The risk stratification of patients with diabetes mellitus (DM) is a major objective for the clinicians, and it can be achieved by coronary flow velocity reserve (CFVR) or with coronary artery calcium score (CS). CS evaluates underlying coronary atherosclerotic plaque burden and CFVR estimates both presence of coronary artery stenosis and microvascular function. Consequently, CFVR may provide unique risk information beyond the extent of coronary atherosclerosis.

Aim: Our aim is to assess joint prognostic value of CFVR and CS in asymptomatic DM patients.

Materials and methods: We prospectively included 200 asymptomatic patients $(45,5 \%$ male, mean age 57,35 $\pm 11,25)$, out of which, there were 101 asymptomatic patients with DM and 99 asymptomatic patients without DM, but with one or more conventionally risk factors for coronary artery disease. We analyzed clinical, biochemical, metabolic, inflammatory parameters, CS by Agatston method, transthoracic Doppler echocardiography CFVR of left anterior descending artery and echocardiographic parameters.

Results: Total CS and CS LAD were significantly higher, while mean CFVR was lower in diabetics compared to the nondiabetics. During 1 year follow-up, 24 patients experienced cardio-vascular events (one cardiovascular death, two strokes, three myocardial infarctions, nine new onsets of unstable angina and nine myocardial revascularizations): 19 patients with DM and five non DM patients, $(p=0,003)$. Overall event free survival was significantly higher in non DM group, compared to the DM group ( $94,9 \%$ vs. $81,2 \%, p=0,002$ respectively), while the patients with CS $\geq 200$ and CFVR $<2$ had the worst outcome during 1 year follow up in the whole study population as well as in the DM group. At multivariable analysis CFVR on LAD (HR 12.918, $95 \% \mathrm{Cl} 3.865-43.177, p<0.001)$ and total CS (HR 13.393, $95 \% \mathrm{Cl}$ $1.675-107.119, p=0.014)$ were independent prognostic predictors of adverse events in DM group of patients.
\end{abstract}

Conclusion: Both CS and CFVR provide independent and complementary prognostic information in asymptomatic DM patients. When two parameters are analyzed together, the risk stratification ability improves, even when DM patients are analyzed together with non DM patients. As a result, DM patients with CS $\geq 200$ and CFVR $<2$ had the worst outcome. Consequently, the use of two tests identified subset of patients who can derive the most benefit from the intensive prevention measures.

Keywords: Coronary artery disease, Calcium score, Coronary flow velocity reserve, Adverse events

\footnotetext{
*Correspondence: miodrag.dikic@gmail.com

${ }^{1}$ Clinic for Cardiology, Clinical Center of Serbia, Visegradska 26, 11000

Belgrade, Serbia

Full list of author information is available at the end of the article
} 


\section{Introduction}

The leading cause of mortality in patients with diabetes is coronary artery disease (CAD). Patients with diabetes mellitus (DM) have increased risk of myocardial infarction and cardiovascular death due to accelerated coronary atherosclerosis, thus the risk stratification of DM patients is a major objective for the clinicians [1]. Traditional cardiovascular risk factors such as age, systolic blood pressure, smoking, cholesterol levels and family history can't completely account for the increased cardiovascular mortality in patients with diabetes [2]. Also novel additional factors like inflammatory factors (fibrinogen, C-reactive protein, Galectin-3) and metabolic factor (lipoproteins) could contribute in explanation of atherosclerosis in diabetes [3-5].

Patients with DM are often asymptomatic until the onset of acute coronary event [6] and they have a poor prognosis [7]. Risk stratification of DM patients with unknown or suspect CAD can be achieved by stress echocardiography [8-13], coronary flow reserve [14-17], or with coronary artery calcium score (CS) $[18,19]$.

Coronary flow velocity reserve (CFVR) reflects both the presence of epicardial coronary artery stenosis and function of microcirculation [20-22]. Impaired CFVR in DM patients is occasionally observed in the absence of significant epicardial coronary arteriosclerosis, even in young adults with DM type I of shorter duration without associated CAD $[8,14,16,22]$.

CS has emerged as promising tool for CAD risk assessment [18]. The amount of CS on cardiac computed tomography has been found to correlate with a total coronary atherosclerotic burden and the risk of adverse cardiovascular outcomes [19]. Recent large prospective study demonstrated that the CS is significant independent predictor of cardiovascular events and can enhance the predictive power of traditional cardiovascular risk factors in asymptomatic patients with diabetes [19]. On the other hand, with the CS result should be cautious since, compared to the computed tomography angiography, it can miss noncalcified plaque components [19].

CS evaluates underlying coronary atherosclerotic plaque burden and CFVR estimates both presence of coronary artery stenosis and microvascular function. Consequently, CFVR may provide unique risk information beyond the extent of coronary atherosclerosis. Thus, our aim is to assess joint prognostic value of CFVR and CS in asymptomatic DM patients.

\section{Methods}

\section{Patients selection}

We prospectively included 200 asymptomatic patients (45,5\% male, mean age 57,35 $\pm 11,25)$, from October 2012 to the March 2013. Out of 200 patients, there were 101 asymptomatic patients with DM diagnosed by standard criteria and on standard diabetic therapy, including diet, tablets, or insulin and 99 asymptomatic patients without $\mathrm{DM}$, but with one or more conventionally risk factors for coronary artery disease.

Patients with a prior history of CAD (including hospital admission for chest pain, acute coronary syndromes, stable angina or prior coronary revascularization) or any other heart disease were excluded from the study in both groups. Also criteria for exclusion from the study were as follows: poor echocardiographic window for CFVR assessment, chronic obstructive pulmonary artery disease, atrioventricular block, inability to provide informed consent.

We pooled biochemical, metabolic parameters (cholesterol, low-density lipoprotein, high-density lipoprotein, triglycerides, glucose level, hemoglobin A1c (HbA1c), microalbuminuria, inflammatory markers of atherosclerosis (high sensitive $C$ reactive protein and fibrinogen). CFVR of left anterior descending artery (LAD) and multi slice computed tomography (MSCT) of coronary arteries with CS calculation according to Agatston method [23] were done in all patients.

\section{Echocardiography and coronary flow velocity reserve}

Echocardiographic studies were performed with an available digital ultrasound system (Acuson Sequoia C256; Siemens Medical Solutions USA, Inc, Mountain View, CA).

To visualize distal part of LAD artery we used modified, foreshortened, three chamber view, obtained by sliding the $3 \mathrm{~V} 2 \mathrm{C}$ multifrequency $(4-\mathrm{MHz})$ transducer on the upper part and medially from an apical three chamber view [20, 24]. For color-Doppler flow mapping, the velocity range was set to the range of $16-24 \mathrm{~cm} / \mathrm{sec}$. Alignment of the ultrasound beam direction with the distal LAD flow was kept as parallel as possible. All the subjects had Doppler recordings of the LAD at rest and during intravenously slow application of adenosine $(0.14 \mathrm{mg} / \mathrm{kg} / \mathrm{min})$, during $2 \mathrm{~min}$. By placing the sample volume on the color signal, spectral Doppler of the LAD showed the characteristic biphasic flow pattern (larger diastolic and smaller systolic components). Coronary diastolic peak velocities were measured at baseline and during the intravenous infusion of adenosine by averaging three consecutive Doppler signals for each measurement. CFVR was defined as ratio of hyperemic and baseline diastolic peak velocities [20,22, 24]. Digital images were obtained and stored on magneto-optical discs and analyzed offline by two well trained physicians.

\section{Calcium score assessment}

All study participants agreed and underwent serial coronary artery CS assessment using MSCT (SOMATOM Sensation 64, Siemens Medical Solutions, Forchheim, Germany) scanner. Heart images view obtained with $100 \mathrm{~ms}$ scan time, using $3 \mathrm{~mm}$ slices, starting at the level 
of carina and proceeding to the level of diaphragm (40-45 slices). Tomography imaging was electrocardiographically triggered at $40 \%$ or $65 \%$ of the R-R interval, depending on the subject's heart rate. CS was defined as a plaque of at least 2 pixels (area $0.67 \mathrm{~mm} 2$ ) with density $\geq 130$ Hounsfield units. Quantitative CS was calculated using Agatston method [23], which involves the multiplication of the area of calcified focus by a cofactor based on the peak density of the lesion. The total CS calculation was done by adding the individual scores of the all lesions found along the entire coronary artery tree [23]. Then, according to the total CS results, patients were grouped into the five CS categories: 0-10, 11-100, 101-400, 401-1000, and $>1000$ Agatston units, as previously used $[19,25]$.

Standard invasive coronary angiography was recommended to selected patients according to the current Guidelines by the referred physician. All diagnostic procedures were done at Clinic for cardiology and Clinic for radiology of Clinical Center of Serbia.

Informed consents were obtained for all the participants; the study was in adherence to the tents of Declaration of Helsinki and the local ethical committee approved the study protocol.

\section{Follow-up data}

During a 1 year follow up of the patients; outcomes were determined from patient interviews at the outpatient clinic, hospital chart reviews, and telephone interviews with patients, their close relatives, or referring physician. Cardiovascular death, stroke, nonfatal myocardial infarction, new onset of unstable angina and clinically driven percutaneous coronary intervention (PCI), or coronary bypass grafting (CABG) were registered as clinical events. Diagnosis of death from cardiovascular causes were obtained from the results of postmortem examination and detailed hospital documentation. Myocardial infarction was defined by typical symptoms, electrocardiographic and cardiac enzyme changes, while stroke was defined as rapid onset of focal or global neurological deficit lasting $\geq 24 \mathrm{~h}$ or leading to death, with clinical findings supplemented by neurological imaging. Unstable angina was diagnosed on the basis of clinical features of an acute coronary syndrome without diagnostic enzyme changes or need for hospital admission or both. Follow-up data were analyzed for the prediction of composite endpoint of major adverse cardiovascular and cerebrovascular events (MACCE).

\section{Statistical analyses}

Descriptive statistics were used to summarize baseline demographic, biochemical and clinical characteristics, and risk factors in two groups - DM and non DM group. Categorical variables were compared using chi-square test. Continuous variables were compared by using Simple $T$ - test, and/or ANOVA-test, or Kruskal Wallis test (for variables without normal distribution). Statistical correlation among CS and CFVR was examined with the Spearman's correlation coefficient. Survival rates were estimated with Kaplan-Meier curves and compared by the log-rank test. The association of selected variables with outcome was assessed with the Cox proportional hazard model using univariate and stepwise multivariate procedures. A significance of 0.05 was required for a variable to be included into the multivariate model, whereas 0.1 was the cut off value for exclusion. Hazard ratios with the corresponding $95 \%$ confidence intervals were estimated. The sensitivity and specificity of CFVR and CS for outcome prediction were evaluated with receiver operating characteristic curves. Analyses were performed using SPSS for Windows version 22 (SPSS, Inc, Chicago, IL).

\section{Results}

Demographic, biochemical and clinical characteristics of the study population were presented in Table 1 . Mean age of the whole study population was $57,7 \pm 11,84$ years $(60,34 \pm 10,21$ years in diabetic and $55,04 \pm 12,81$ years in non diabetic group, $p<0.001)$. Subjects were obese with mean body mass index $27,39 \pm 4,73 \mathrm{~kg} / \mathrm{m}^{2}(28,74 \pm$ $4,68 \mathrm{~kg} / \mathrm{m}^{2}$ in DM group and $26,01 \pm 4,39 \mathrm{~kg} / \mathrm{m}^{2}$ in non DM group, $p<0.001)$. Average systolic blood pressure for the whole population was $128,03 \pm 11,44 \mathrm{mmHg}$ $(130,10 \pm 11,27 \mathrm{mmHg}$ in DM group and 125,91 \pm $11,28 \mathrm{mmHg}$ in non DM group, $p=0,009$ ).

There were no significant differences between DM and non DM group in gender, prevalence of smoking and family history of cardiovascular disease, level of cholesterol, low-density lipoproteins, high sensitive C-reactive protein and fibrinogen level (Table 1). Diabetic patients had significantly more often hypertension, microalbuminuria, higher systolic blood pressure, lower level of HDL, higher level of triglycerides, glucose, HbA1C (Table 1).

CFVR and CS parameters were presented in Table 2. There were no differences between groups in baseline and hyperemic velocities of $\mathrm{LAD}$, but mean value of CFVR was significantly lower in DM group $(p=0,011)$. The percent of patients with pathological values of CFVR $<2$ was significantly higher in diabetics compared to the nondiabetics (18,8 \% vs. $3,0 \%, p<0,001$, respectively). Total CS and CS LAD were significantly higher in diabetics compared to the non DM group. In our study there was significantly higher rate of CS $0-100$ in non diabetics $(74,8 \%$ vs. $44,6 \%$ ), while higher rate of CS $\geq 401$ was found in diabetics (20,8 \% vs. $5,0 \%)$. In subgroup of patients with cut off of CS $\geq 200$ Agatston units there was significantly higher rate of diabetics compared to non diabetics (44,6\% vs. $15,2 \%, p<0,001$ respectively). 
Table 1 Demographic, biochemical and clinical characteristics of the study population

\begin{tabular}{|c|c|c|c|c|c|}
\hline \multirow[t]{2}{*}{ Variables } & & \multirow{2}{*}{$\begin{array}{l}\text { Non DM } \\
(n=99)\end{array}$} & \multirow{2}{*}{$\begin{array}{l}\mathrm{DM} \\
(n=101)\end{array}$} & \multirow{2}{*}{$\begin{array}{l}\text { Total } \\
(n=200)\end{array}$} & \multirow{2}{*}{$\begin{array}{l}p \text { value: non } \\
\text { DM vs. DM }\end{array}$} \\
\hline & & & & & \\
\hline Male, no. (\%) & & $42(42,4 \%)$ & $49(48,5 \%)$ & $91(45,5 \%)$ & $p=0,387$ \\
\hline Age & & $55,04 \pm 12,81$ & $60,34 \pm 10,21$ & $57,7 \pm 11,84$ & $p<0,001$ \\
\hline \multirow[t]{2}{*}{ Obesity } & $\mathrm{BMI} \leq 25$ & $52(52,5 \%)$ & $20(19,8 \%)$ & $72(36,0 \%)$ & \multirow[t]{2}{*}{$p<0,001$} \\
\hline & $\mathrm{BMI}>25$ & $47(47,5 \%)$ & $81(80,2 \%)$ & $128(64,0 \%)$ & \\
\hline BMI (kg/m2) & & $26,01 \pm 4,39$ & $28,74 \pm 4,68$ & $27,39 \pm 4,73$ & $p<0,001$ \\
\hline \multirow[t]{3}{*}{ Smoking History, no. (\%) } & Current & $28(28,2 \%)$ & $19(18,8 \%)$ & $47(23,5 \%)$ & \multirow[t]{3}{*}{$p=0,261$} \\
\hline & Former & $16(16,2 \%)$ & $21(20,8 \%)$ & $37(18,5 \%)$ & \\
\hline & Non smoker & $55(55,56 \%)$ & $61(60,40 \%)$ & $116(58,00 \%)$ & \\
\hline \multicolumn{2}{|l|}{ Family history, no. (\%) } & $65(65,70 \%)$ & $70(69,30 \%)$ & $135(67,50 \%)$ & $p=0,582$ \\
\hline \multicolumn{2}{|l|}{ HTA, no. (\%) } & $54(55,70 \%)$ & $86(85,10 \%)$ & $140(70,70 \%)$ & $p<0,001$ \\
\hline \multicolumn{2}{|c|}{ Systolic blood pressure (mmHg) } & $125,91 \pm 11,28$ & $130,10 \pm 11,27$ & $128,03 \pm 11,44$ & $p=0,009$ \\
\hline \multicolumn{2}{|c|}{ Hypercholesterolemia, no. (\%) } & $52(53,1 \%)$ & $72(72 \%)$ & $124(62,6 \%)$ & $p=0,006$ \\
\hline \multicolumn{2}{|l|}{ Cholesterol (mmol/l) } & $5,54 \pm 0,93$ & $5,36 \pm 0,91$ & $5,45 \pm 0,92$ & $p=0,160$ \\
\hline \multicolumn{2}{|l|}{ LDL (mmol/l) } & $3,40 \pm 0,87$ & $3,26 \pm 0,81$ & $3,33 \pm 0,84$ & $p=0,253$ \\
\hline \multicolumn{2}{|l|}{$\mathrm{HDL}(\mathrm{mmol} / \mathrm{l})$} & $1,38 \pm 0,33$ & $1,19 \pm 0,26$ & $1,28 \pm 0,31$ & $p<0,001$ \\
\hline \multicolumn{2}{|l|}{ Triglycerides (mmol/l) } & $1,52 \pm 0,80$ & $2,10 \pm 1,12$ & $1,82 \pm 1,02$ & $p<0,001$ \\
\hline \multicolumn{2}{|l|}{ Glucose (mmol/l) } & $5,37 \pm 0,74$ & $8,31 \pm 2,76$ & $6,86 \pm 2,51$ & $p<0,001$ \\
\hline \multicolumn{2}{|l|}{$\mathrm{HbA1C}(\%)$} & $5,51 \pm 0,81$ & $7,13 \pm 1,32$ & $6,33 \pm 1,36$ & $p<0,001$ \\
\hline \multicolumn{2}{|l|}{ CRP (mg/l) } & $2,13 \pm 1,71$ & $2,89 \pm 3,47$ & $2,51 \pm 2,77$ & $p=0,141$ \\
\hline \multicolumn{2}{|l|}{ Fibrinogen (g/l) } & $3,63 \pm 1,04$ & $3,58 \pm 1,05$ & $3,61 \pm 1,05$ & $p=0,734$ \\
\hline \multicolumn{2}{|l|}{ Microalbuminuria, no. (\%) } & $0(0,00 \%)$ & $26(25,7 \%)$ & $26(13,0 \%)$ & $p<0,001$ \\
\hline
\end{tabular}

Plus-minus values are means $\pm S D, B M I$ body mass index, $C R P$ C-reactive protein, $D M$ diabetes mellitus, $L D L$ low-density lipoprotein, $H D L$ high-density lipoprotein, HbA1C hemoglobin A1c, HTA hypertension

There were significant correlations between CFVR LAD and total CS $(r=-0,266, p=0.008)$ as well as with CS of LAD artery $(r=-0,249, p=0.013)$ in DM patients. CFVR and CS did not correlate significantly in non DM patients. There was also no relation between HbA1c and CFVR of LAD in our DM population.

During 1 year follow-up, 24 patients (12\%) experienced cardio-vascular events: 19 patients with DM $(17,8 \%)$ and five patients $(5,1 \%)$ in non DM group, $(p=0,003)$, Table 3.

When we analyzed type of events, there were significantly higher number of PCIs in diabetic group compared to non diabetic group ( $31,6 \%$ vs. $20 \%, p=0,050$ respectively). There were no significant differences in death, MI, CABG and onset of angina between two groups. Using a receiver operating characteristic analysis, CFVR $=2$ and $C S=200$ were the best predictors of future events (area under the curve $=0,801$, sensitivity $=66,7 \%$, specificity $=83,0 \%$ and area under the curve $=0,928$, sensitivity $=91,7 \%$, specificity $=79,2 \%$, respectively) and were taken as cut off values for the further statistics.

Overall event free survival was significantly higher in non DM group, compared to the DM group (94,9\% vs. $81,2 \%, p=0,002$ respectively),as presented in Fig. 1, while the patients with CS $\geq 200$ and CFVR $<2$ had the worst outcome during 1 year follow up in the whole study population (Fig. 2), as well as in the DM group (Fig. 3).

In the whole study population patients with CS $\geq 200$ and CFVR $<2$ had a 24,3 fold increase in cardiovascular risk compared to the patients with CS $<200$ and CFVR $\geq 2$ (95\% CI: $5,13-106,97 ; p<0.001$ ), while in the DM group, patients with CS $\geq 200$ and CFVR $<2$ had 43,7 fold increase in cardiovascular risk compared to the patients with CS $<200$ and CFVR $\geq 2$ (95 \% CI: 7,09-269,77; $p<0,001$ ).

Univariate predictors of MACCE are reported in Table 4. At multivariable analysis CFVR on LAD (HR 12.918, $95 \%$ CI 3.865-43.177, $p<0.001$ ) and total CS (HR 13.393, $95 \%$ CI 1.675-107.119, $p=0.014$ ) were independent prognostic predictors of MACCE in DM group of patients as presented in Table 4.

\section{Discussion}

This study, represents the results of the annual follow up regarding the prognostic value of CS and CFVR in asymptomatic diabetic patients, where abnormal CFVR $<2$, detected by Doppler echocardiography identified together with CS $\geq 200$ subset of patients at higher risk for adverse 
Table 2 Coronary flow velocity reserve and calcium score parameters

\begin{tabular}{|c|c|c|c|c|c|}
\hline \multirow{2}{*}{\multicolumn{2}{|c|}{ Variables }} & Non DM & DM & Total & \multirow{2}{*}{$\begin{array}{l}p \text { value: nor } \\
\text { DM vs. DM }\end{array}$} \\
\hline & & $(n=99)$ & $(n=101)$ & $(n=200)$ & \\
\hline \multicolumn{2}{|c|}{ Baseline diastolic flow velocity (m/sec), LAD } & $0,27 \pm 0,05$ & $0,27 \pm 0,07$ & $0,27 \pm 0,06$ & $p=0,663$ \\
\hline \multicolumn{2}{|c|}{ Hyperemic diastolic flow velocity (m/sec), LAD } & $0,72 \pm 0,16$ & $0,67 \pm 0,19$ & $0,70 \pm 0,18$ & $p=0,061$ \\
\hline \multicolumn{2}{|c|}{ Rest heart rate } & $71,27 \pm 10,86$ & $70,70 \pm 9,11$ & $70,98 \pm 10,0$ & $p=0,687$ \\
\hline \multicolumn{2}{|c|}{ Hyperemic heart rate } & $76,23 \pm 12,36$ & $75,23 \pm 10,82$ & $76,06 \pm 11,58$ & $p=0,835$ \\
\hline \multicolumn{2}{|l|}{ CFVR } & $2,65 \pm 0,41$ & $2,48 \pm 0,44$ & $2,56 \pm 0,44$ & $p=0,011$ \\
\hline \multicolumn{2}{|l|}{ CFVR $<2$} & $3(3,0 \%)$ & $19(18,8 \%)$ & $22(11,0 \%)$ & $p<0,001$ \\
\hline \multicolumn{2}{|l|}{$C F V R \geq 2$} & $96(97,0 \%)$ & $82(81,2 \%)$ & $178(89,0 \%)$ & \\
\hline \multicolumn{2}{|l|}{ CS - total } & $93,10 \pm 196,59$ & $322,76 \pm 534,84$ & $209,09 \pm 419,56$ & $p<0,001$ \\
\hline \multicolumn{2}{|l|}{$C S-L A D$} & $46,16 \pm 93,24$ & $142,32 \pm 232,88$ & $96,24 \pm 184,40$ & $p<0,001$ \\
\hline \multirow[t]{5}{*}{ CS, no. (\%) } & $0-10$ & $56(56,6 \%)$ & $31(30,7 \%)$ & 87 (43,5 \%) & $p<0,001$ \\
\hline & $11-100$ & $18(18,2 \%)$ & $14(13,9 \%)$ & $32(16,0 \%)$ & \\
\hline & $101-400$ & $20(20,2 \%)$ & $35(34,6 \%)$ & $55(27,5 \%)$ & \\
\hline & $401-1000$ & $3(3,0 \%)$ & $14(13,9 \%)$ & 17 (8,5 \%) & \\
\hline & $\geq 1001$ & $2(2,0 \%)$ & $7(6,9 \%)$ & $9(4,5 \%)$ & \\
\hline \multicolumn{2}{|l|}{ CS 0-199 } & $84(84,8 \%)$ & $56(55,4 \%)$ & $140(70,0 \%)$ & $p<0,001$ \\
\hline \multicolumn{2}{|l|}{$C S \geq 200$} & 15 (15,2 \%) & 45 (44,6 \%) & 60 (30,0 \%) & \\
\hline
\end{tabular}

Plus-minus values are means $\pm S D, D M$ diabetes mellitus, LAD left anterior descending artery, CS calcium score, CFVR coronary flow velocity reserve, CVD cardiovascular disease

Table 3 Major adverse cardiac and cerebrovascular events, calcium score and coronary flow velocity reserve in patients with and without diabetes mellitus

\begin{tabular}{|c|c|c|c|c|c|c|c|c|}
\hline & & \multirow{2}{*}{\multicolumn{2}{|c|}{$\begin{array}{l}\text { Non DM } \\
(n=99)\end{array}$}} & \multirow{2}{*}{\multicolumn{2}{|c|}{$\begin{array}{l}\text { DM } \\
(n=101)\end{array}$}} & \multirow{3}{*}{$\begin{array}{l}\text { Total } \\
(n=200) \\
\text { Number }\end{array}$} & \multirow[t]{3}{*}{$\%$} & \multirow{3}{*}{$\begin{array}{l}p \text { value: nor } \\
\text { DM vs DM }\end{array}$} \\
\hline & & & & & & & & \\
\hline & & Number & $\%$ & Number & $\%$ & & & \\
\hline \multirow[t]{2}{*}{ MACCE } & No & 94 & $94,9 \%$ & 82 & $81,2 \%$ & 176 & $88 \%$ & $p=0,003$ \\
\hline & Yes & 5 & $5,1 \%$ & 19 & $18,8 \%$ & 24 & $12 \%$ & \\
\hline \multirow[t]{2}{*}{ CS/MACCE } & 0-199 & 1 & $20 \%$ & 1 & $5,3 \%$ & 2 & $8,3 \%$ & $p=0,289$ \\
\hline & $\geq 200$ & 4 & $80 \%$ & 18 & $94,7 \%$ & 22 & $91,7 \%$ & \\
\hline \multirow[t]{2}{*}{ CFVR/MACCE } & CFVR $<2$ & 1 & $20 \%$ & 15 & $78,9 \%$ & 16 & $66,7 \%$ & $p=0,013$ \\
\hline & CFVR $\geq 2$ & 4 & $80 \%$ & 4 & $21.1 \%$ & 8 & $33,3 \%$ & \\
\hline \multirow[t]{6}{*}{ Events } & Death & 0 & $0,0 \%$ & 1 & $5,3 \%$ & 1 & $4,2 \%$ & $p=0,321$ \\
\hline & $\mathrm{Ml}$ & 1 & $20 \%$ & 2 & $10,5 \%$ & 3 & $12,5 \%$ & $p=0,573$ \\
\hline & $\mathrm{PCl}$ & 1 & $20 \%$ & 6 & $31,6 \%$ & 7 & $29,2 \%$ & $p=0,050$ \\
\hline & CABG & 0 & $0,0 \%$ & 2 & $10,5 \%$ & 2 & $8,3 \%$ & $p=0,159$ \\
\hline & Stroke & 1 & $20 \%$ & 1 & $5,3 \%$ & 2 & $8,3 \%$ & $p=0,989$ \\
\hline & Unstable Angina & 2 & $40 \%$ & 7 & $36,8 \%$ & 9 & $37,5 \%$ & $p=0,987$ \\
\hline \multirow{2}{*}{\multicolumn{2}{|c|}{ Mean follow-up -months }} & \multicolumn{2}{|c|}{$15,44 \pm 3,38$} & \multicolumn{2}{|c|}{$14,14 \pm 5,95$} & & & \\
\hline & & \multicolumn{2}{|c|}{$(14,46-17,84)$} & \multicolumn{2}{|c|}{$(12,96-15,32)$} & & & \\
\hline \multicolumn{2}{|l|}{ Overall survival } & \multicolumn{2}{|c|}{$94,9 \% \pm 2,2 \%$} & \multicolumn{2}{|c|}{$81,2 \% \pm 3,8 \%$} & & & \\
\hline
\end{tabular}

Plus-minus values are means \pm SD, CS calcium score, CFVR coronary flow velocity reserve, DM diabetes mellitus, MACCE major adverse cardiac and cerebrovascular event, $M I$ myocardial infarction, $P C I$ percutaneous coronary intervention, $C A B G$ coronary arteries bypass grafting 


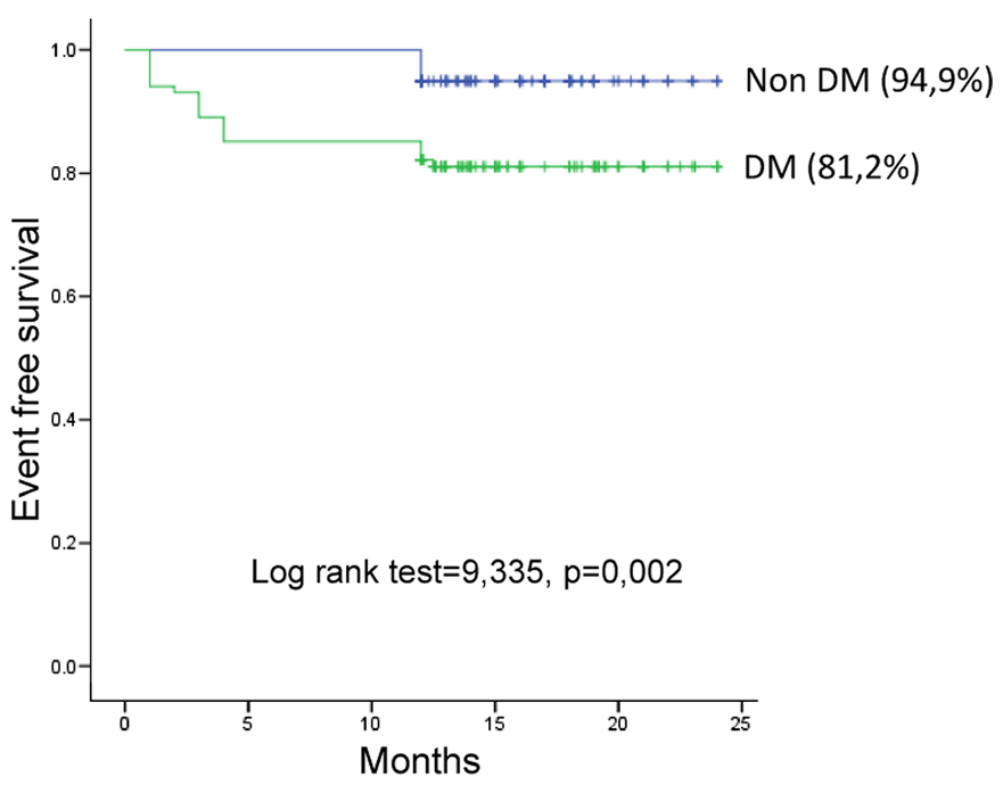

Fig. 1 Kaplan-Meier event-free survival curve in patients with and without diabetes mellitus. DM: diabetes mellitus

events. When the two parameters are analyzed together, the risk stratification ability improves, even when DM patients are analyzed together with non DM patients. Thus, our data confirms and expands previous studies, suggesting not only that CFVR and CS have prognostic value when separately analyzed but also that the combination of the two parameters has additive value and that they are complementary in their power of prediction. In our study, we found that older male as well as obese patients, mostly diabetics, have higher level of CS. At the same time, asymptomatic diabetic patients had significantly higher both total CS and CS of LAD artery compared to the control group which is consistent with published studies [25-27].

The presence of diabetes carries a higher risk, and studies have shown that CS 0 can be helpful to stratificate these subjects into the low-risk category, with a lower rate of adverse events and excellent survival. In asymptomatic individuals with DM, prevalence of CS 0 in patients without diabetes was twice as high as compared to the subjects with diabetes with the same CS, as similar to our study, but there was no difference in survival during 5 years follow up $(98.8 \%$ vs. $99.4 \%, p=0,5)$

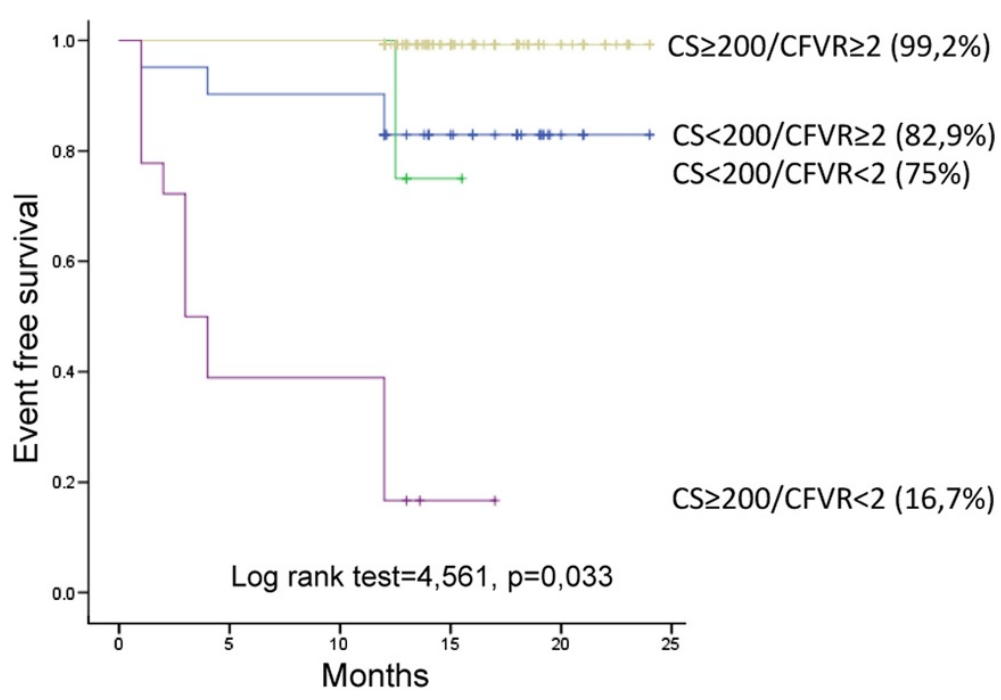

Fig. 2 Kaplan-Meier event-free survival curve in diabetic and non diabetic patients stratified according to the presence of CS $<200$ or CS $\geq 200$ and CFVR $<2$ or CFVR $\geq 2$. The worst survival is observed in patients with CS $\geq 200$ and CFVR $<2$. CS: calcium score, CFVR: coronary flow velocity reserve 


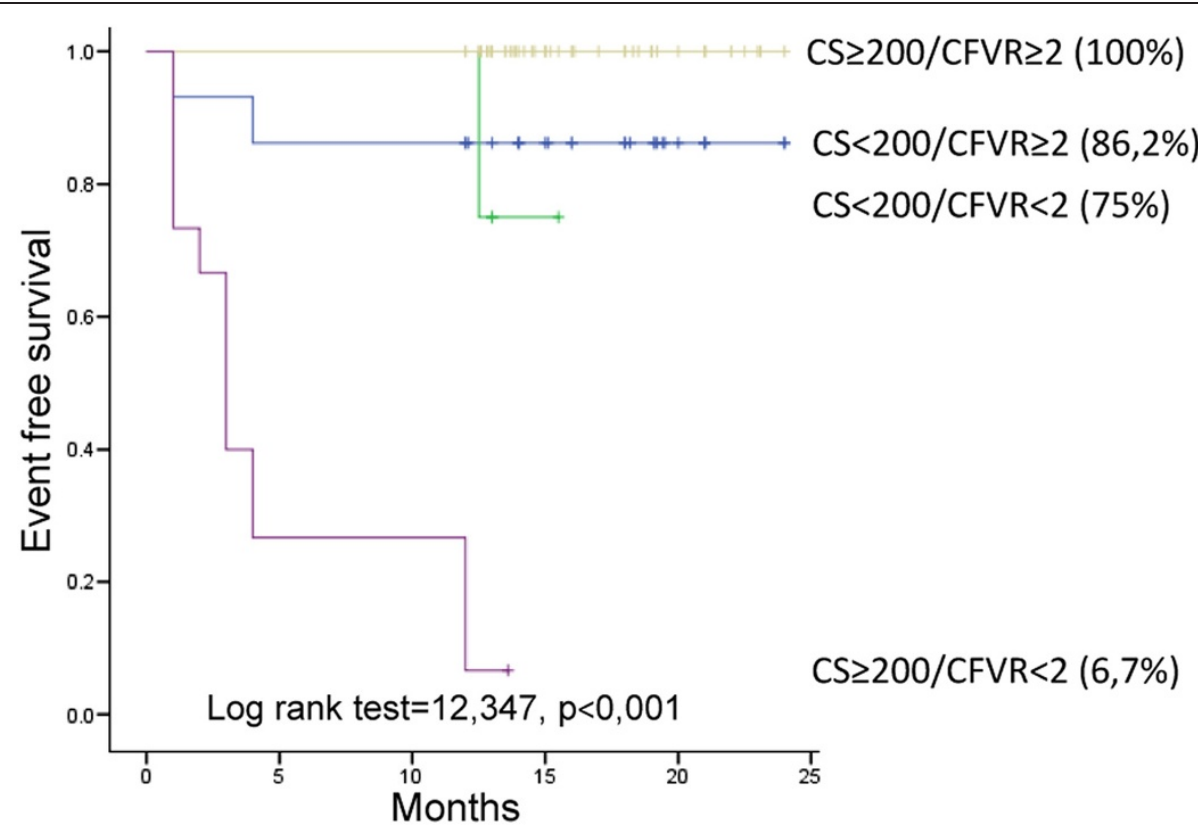

Fig. 3 Kaplan-Meier event-free survival curve in diabetic patients stratified according to the presence of CS $<200$ or CS $\geq 200$ and CFVR $<2$ or $C F V R \geq 2$. The worst survival is observed in patients with CS $\geq 200$ and CFVR $<2$. CS: calcium score, CFVR: coronary flow velocity reserve

[25]. Meta-analysis of 18 studies showed that CS 0 had a negative predictive value of $93 \%$ for the presence of obstructive CAD, suggesting that CS 0 can safely exclude presence of obstructive CAD [28]. Other studies also showed that patients with $C S=0$, have very low risk of cardiac events during the follow-up as well as in our study $[29,30]$. In the study with asymptomatic DM patients during 8-years follow-up, it was presented that the CS over 400 had a significantly higher prevalence of cardiovascular events compared to the group with lower CS (5.6 \% vs. $0.7 \%, p<0.01)$ [31]. It was also shown, that CS increased in proportion to an adverse events from 0 to $18 \%$ when the score rised from 100 to 1000 Agatston units [31]. We presented that diabetics with CS $\geq 200$ had more often MACCE, which is consistent with the results of published studies [31, 32]. Prediction of adverse cardiovascular events in asymptomatic DM patients compared according to the value of CS and

Table 4 Univariate and multivariate prognostic predictors of adverse events in diabetic patient

\begin{tabular}{|c|c|c|c|c|c|c|c|c|}
\hline & \multicolumn{4}{|c|}{ Univariate analysis } & \multicolumn{4}{|c|}{ Multivariate analysis } \\
\hline & \multirow[t]{2}{*}{$\mathrm{HR}$} & \multicolumn{2}{|c|}{$95.0 \% \mathrm{Cl}$ for HR } & \multirow[t]{2}{*}{$p$} & \multirow[t]{2}{*}{$\mathrm{HR}$} & \multicolumn{2}{|c|}{$95.0 \% \mathrm{Cl}$ for $\mathrm{HR}$} & \multirow[t]{2}{*}{$p$} \\
\hline & & Lower & Upper & & & Lower & Upper & \\
\hline Gender (male) & 4,365 & 1,448 & 13,162 & 0,009 & 1,431 & 0,455 & 4,498 & 0,539 \\
\hline Age & 1,863 & 0,671 & 5,173 & 0,233 & & & & \\
\hline Obesity & 0,970 & 0,322 & 2,925 & 0,958 & & & & \\
\hline HTA & 1,602 & 0,370 & 6,937 & 0,528 & & & & \\
\hline Family history of CAD & 0,948 & 0,360 & 2,494 & 0,913 & & & & \\
\hline Smoking habit & 0,891 & 0,351 & 2,263 & 0,808 & & & & \\
\hline Hypercholesterolemia & 2,533 & 1,028 & 6,239 & 0,043 & 1,149 & 0,441 & 2,990 & 0,777 \\
\hline Microalbuminuria & 1,713 & 0,674 & 4,351 & 0,258 & & & & \\
\hline CRP & 1,503 & 0,604 & 3,736 & 0,381 & & & & \\
\hline Fibrinogen & 1,100 & 0,447 & 2,706 & 0,863 & & & & \\
\hline CS $(\geq 200<)$ & 26,707 & 3,559 & 200,381 & 0,001 & 13,393 & 1,675 & 107,119 & 0,014 \\
\hline CFVR $(\geq 2<)$ & 24,240 & 7,944 & 73,970 & $<0,001$ & 12,918 & 3,865 & 43,177 & $<0,001$ \\
\hline
\end{tabular}

CRP C-reactive protein, HTA hypertension, CS calcium score, CFVR coronary flow velocity reserve, CAD coronary artery disease, $H R$ hazard ratio, $C I$ confidence interval 
followed during 4 years, was recently presented in prospective cohort study [19]. Authors analyzed classical risk factors, as well as metabolic and inflammatory factors (lipoprotein, apolipoprotein, homocysteine and C-reactive protein) [19]. They showed that the CS is independent predictor of the cardiovascular events [19]. We also analyzed inflammation (CRP and fibrinogen), and metabolic (glycated hemoglobin, hypercholesterolemia and microalbuminuria) parameters but we found that the CFVR and CS were the only independent predictors of adverse events.

As previously showed, we found that CFVR LAD was significantly lower in DM group compared to the non DM group $[14,15,22]$. Nemes et al. determined the prognostic impact of diabetes and CFVR in patients with suspected coronary artery disease. During $41 \pm 12$ months they recorded 22 cardiovascular deaths (13 sudden cardiac death, seven acute heart failure, two had cardiovascular thrombosis) [17]. Multivariable regression analysis showed that only CFVR and DM were independent predictors of cardiovascular survival [17]. According to the ROC analysis, the best predictive value of CFVR was 1.73 [17]. In contrast to this study, our study examined asymptomatic patients with and without diabetes and had only one death of DM patient during the shorter follow up, while the best predictor value of CFVR for the adverse events was two. Cortigiani et al. furthermore point out importance of evaluation of microcirculation in DM patients without severe CAD $(\leq 50 \%$ diameter coronary stenosis), where CFVR $\leq 2$ represented a strong and independent predictor of the combined event of death and nonfatal MI predicting a nearly seven times higher yearly hard-event rate compared with preserved CFVR [14]. In the same study, nonobstructive CAD failed to provide independent prognostic contribution, although it was associated with significantly lower mean CFVR [14].

Reduced CFVR in DM patients without coronary artery disease is due to functional and structural changes of coronary microcirculation [33-35]. It is also known that coronary microcirculatory dysfunction can reduce CFVR, even without inducing regional wall motion abnormalities or before the occurrence of coronary artery stenosis, but can still be linked with adverse events in patients with DM or LV hypertrophy [8, 9, 14, 24]. Microvascular dysfunction affects LV globally [14], thus the CFVR LAD is excellent option for evaluation of microcirculation due to high feasibility (94-98 \%) in various studies [8, 14, 20, 24]. Therefore we found that in asymptomatic DM patients the assessment of CFVR as a marker of function of both micro- and macro-circulation together with evaluation of coronary morphology by CS is a reasonable diagnostic method for the risk stratification of adverse coronary events.

\section{Study limitations}

There were some limitations in our study. First we enrolled asymptomatic diabetic patients, and asymptomatic controls according to their anamnestic data that they don't have any anginal symptoms and fatigue. Our study is relatively small, because there were just 200 subjects enrolled out of which $101 \mathrm{DM}$ patients. Also we did not have age matched control group, but we believe that eventhough the age was significantly different between groups that difference cannot affect significantly values of CFVR and CS [36]. Although measurement of CFVR in all three coronary arteries is preferable, CFVR was measured only in the LAD due to technical challenge in the present time to visualize all three coronary arteries with high feasibility [24]. We didn't focus on diastolic dysfunction, which is reported in diabetes, and leads to the impaired CFVR as well as hypertension. However in both groups we had patients with hypertension, which contributed to the results of CFVR in both groups.

\section{Conclusion}

Both CS and CFVR obtained by MSCT and by transthoracic Doppler echocardiography assessments, respectively, provide independent and complementary prognostic information in asymptomatic DM patients. When the two parameters are analyzed together, the risk stratification ability improves, even when DM patients are analyzed together with non DM patients. As a result, DM patients with CS $\geq 200$ and CFVR $<2$ had the worst outcome. Consequently, the use of two tests identified subset of patients who can derive the most benefit from the intensive prevention measures - more aggressive control of the risk factors and more frequent follow-up by noninvasive testing.

\section{Abbreviations \\ DM: Diabetes mellitus; non DM: Control group; AS: Agatston score; MACCE: Major adverse cardiovascular and cerebrovascular events; HTA: Hypertension; CRP: C-reactive protein; CAD: Coronary artery disease; CFVR: Coronary flow velocity reserve; Cl: Confidence interval; CS: Calcium score; LAD: Left anterior descending coronary artery; MSCT: Multidetector computed tomography; MI: Myocardial infarction; PCI: Percutaneous coronary intervention; CABG: Coronary bypass grafting.}

\section{Competing interests}

The authors declare that they have no competing interests.

\section{Authors' contributions}

MD participated in the writing of the manuscript and acquisition and analysis of the data. MT performed CFVR, as well as participated in statistical analysis and writing of the manuscript. ZM and AM performed all CS examinations and participated in the study design. VG and IJ performed echocardiographic and clinical examinations. ADj-D performed CFVR. JSt participated in the manuscript preparation and echocardiographic examination. JSe performed clinical examinations and recruitment of the patients. MO participated in the conception and design of the study. BB did Cath Lab examinations and helped preparation of the manuscript. AA participated in study design and coordination. All authors read and approved the final manuscript.

\section{Author details}

${ }^{1}$ Clinic for Cardiology, Clinical Center of Serbia, Visegradska 26, 11000 Belgrade, Serbia. ${ }^{2}$ Medical School, University of Belgrade, Belgrade, Serbia. 
${ }^{3}$ Clinic for Radiology, Clinical Center of Serbia, Belgrade, Serbia. ${ }^{4}$ Clinic for Endocrinology, Clinical Center of Serbia, Belgrade, Serbia. ${ }^{5}$ Cardiology Department, Clinical Hospital Zvezdara, Belgrade, Serbia.

Received: 23 June 2015 Accepted: 2 September 2015 Published online: 04 September 2015

\section{References}

1. Mazzone T. Reducing cardiovascular disease in patients with diabetes mellitus. Curr Opin Cardiol. 2005;20(4):245-9.

2. Saito I, Folsom AR, Brancati FL, Duncan BB, Chambless LE, McGovern PG. Nontraditional risk factors for coronary heart disease incidence among persons with diabetes: the Atherosclerosis Risk in Communities (ARIC) Study. Ann Intern Med. 2000;133(2):81-91.

3. Mazzone T. Strategies in ongoing clinical trials to reduce cardiovascular disease in patients with diabetes mellitus and insulin resistance. Am J Cardiol. 2004;93(11A):27C-31C.

4. Grundy SM, Benjamin IJ, Burke GL, Chait A, Eckel RH, Howard BV, et al. Diabetes and cardiovascular disease: a statement for healthcare professionals from the American Heart Association. Circulation. 1999;100(10):1134-46.

5. Seferovic JP, Lalic NM, Floridi F, Tesic M, Seferovic PM, Giga V, et al. Structural myocardial alterations in diabetes and hypertension: the role of galectin-3. Clin Chem Lab Med. 2014;52(10):1499-505.

6. Alexander CM, Landsman PB, Teutsch SM. Diabetes mellitus, impaired fasting glucose, atherosclerotic risk factors, and prevalence of coronary heart disease. Am J Cardiol. 2000;86(9):897-902.

7. Wannamethee SG, Shaper AG, Lennon L. Cardiovascular disease incidence and mortality in older men with diabetes and in men with coronary heart disease. Heart. 2004;90(12):1398-403.

8. Cortigiani L, Rigo F, Gherardi S, Sicari R, Galderisi M, Bovenzi F, et al. Additional prognostic value of coronary flow reserve in diabetic and nondiabetic patients with negative dipyridamole stress echocardiography by wall motion criteria. J Am Coll Cardiol. 2007;50(14):1354-61.

9. Rigo F, Cortigiani L, Pasanisi E, Richieri M, Cutaia V, Celestre M, et al. The additional prognostic value of coronary flow reserve on left anterior descending artery in patients with negative stress echo by wall motion criteria. A Transthoracic Vasodilator Stress Echocardiography Study. Am Heart J. 2006;151(1):124-30.

10. Marwick TH, Case C, Sawada S, Vasey C, Short L, Lauer M. Use of stress echocardiography to predict mortality in patients with diabetes and known or suspected coronary artery disease. Diabetes Care. 2002;25(6):1042-8.

11. Elhendy A, Arruda AM, Mahoney DW, Pellikka PA. Prognostic stratification of diabetic patients by exercise echocardiography. J Am Coll Cardiol. 2001;37(6):1551-7.

12. Kamalesh M, Matorin R, Sawada S. Prognostic value of a negative stress echocardiographic study in diabetic patients. Am Heart J. 2002;143(1):163-8.

13. Sozzi FB, Elhendy A, Roelandt JR, van Domburg RT, Schinkel AF, Vourvouri $\mathrm{EC}$, et al. Prognostic value of dobutamine stress echocardiography in patients with diabetes. Diabetes Care. 2003;26(4):1074-8.

14. Cortigiani L, Rigo F, Gherardi S, Galderisi M, Bovenzi F, Sicari R. Prognostic meaning of coronary microvascular disease in type 2 diabetes mellitus: a transthoracic Doppler echocardiographic study. J Am Soc Echocardiogr. 2014;27(7):742-8.

15. Dayanikli F, Grambow D, Muzik O, Mosca L, Rubenfire M, Schwaiger M. Early detection of abnormal coronary flow reserve in asymptomatic men at high risk for coronary artery disease using positron emission tomography. Circulation. 1994;90(2):808-17.

16. Pitkanen OP, Nuutila P, Raitakari OT, Ronnemaa T, Koskinen PJ, lida H, et al. Coronary flow reserve is reduced in young men with IDDM. Diabetes. 1998:47(2):248-54.

17. Nemes A, Forster T, Geleijnse ML, Kutyifa V, Neu K, Soliman Ol, et al. The additional prognostic power of diabetes mellitus on coronary flow reserve in patients with suspected coronary artery disease. Diabetes Res Clin Pract. 2007;78(1):126-31.

18. Greenland P, Bonow RO, Brundage BH, Budoff MJ, Eisenberg MJ, Grundy SM, et al. ACCF/AHA 2007 clinical expert consensus document on coronary artery calcium scoring by computed tomography in global cardiovascular risk assessment and in evaluation of patients with chest pain: a report of the American College of Cardiology Foundation Clinical Expert Consensus Task Force (ACCF/AHA Writing Committee to Update the 2000 Expert
Consensus Document on Electron Beam Computed Tomography). Circulation. 2007;115(3):402-26.

19. Elkeles RS, Godsland IF, Feher MD, Rubens MB, Roughton M, Nugara F, et al. Coronary calcium measurement improves prediction of cardiovascular events in asymptomatic patients with type 2 diabetes: the PREDICT study. Eur Heart J. 2008;29(18):2244-51.

20. Tesic M, Djordjevic-Dikic A, Beleslin B, Trifunovic D, Giga V, Marinkovic J, et al. Regional difference of microcirculation in patients with asymmetric hypertrophic cardiomyopathy: transthoracic Doppler coronary flow velocity reserve analysis. J Am Soc Echocardiogr. 2013;26(7):775-82.

21. Kostic J, Djordjevic-Dikic A, Dobric M, Milasinovic D, Nedeljkovic M, Stojkovic $S$, et al. The effects of nicorandil on microvascular function in patients with ST segment elevation myocardial infarction undergoing primary PCl. Cardiovasc Ultrasound. 2015;13:26.

22. Dimitrow PP, Galderisi M, Rigo F. The non-invasive documentation of coronary microcirculation impairment: role of transthoracic echocardiography. Cardiovasc Ultrasound. 2005;3:18

23. Agatston AS, Janowitz WR, Hildner FJ, Zusmer NR, Viamonte Jr M, Detrano R. Quantification of coronary artery calcium using ultrafast computed tomography. J Am Coll Cardiol. 1990;15(4):827-32.

24. Rigo F. Coronary flow reserve in stress-echo lab. From pathophysiologic toy to diagnostic tool. Cardiovasc Ultrasound. 2005;3:8.

25. Raggi P, Shaw LJ, Berman DS, Callister TQ. Prognostic value of coronary artery calcium screening in subjects with and without diabetes. J Am Coll Cardiol. 2004;43(9):1663-9.

26. Hoff JA, Quinn L, Sevrukov A, Lipton RB, Daviglus M, Garside DB, et al. The prevalence of coronary artery calcium among diabetic individuals without known coronary artery disease. J Am Coll Cardiol. 2003;41(6):1008-12.

27. Schurgin S, Rich $S$, Mazzone T. Increased prevalence of significant coronary artery calcification in patients with diabetes. Diabetes Care. 2001;24(2):335-8.

28. Sarwar A, Shaw L, Shapiro MD, Blankstein R, Hoffmann U, Cury RC, et al. Diagnostic and prognostic value of absence of coronary artery calcification. JACC Cardiovasc Imaging. 2009;2(6):675-88.

29. Budoff MJ, Shaw L, Liu ST, Weinstein SR, Mosler TP, Tseng PH, et al. Long-term prognosis associated with coronary calcification: observations from a registry of 25,253 patients. J Am Coll Cardiol. 2007;49(18):1860-70.

30. Detrano R, Guerci AD, Carr JJ, Bild DE, Burke G, Folsom AR, et al. Coronary calcium as a predictor of coronary events in four racial or ethnic groups. $N$ Engl J Med. 2008;358(13):1336-45.

31. Becker A, Leber AW, Becker C, von Ziegler F, Tittus J, Schroeder I, et al. Predictive value of coronary calcifications for future cardiac events in asymptomatic patients with diabetes mellitus: a prospective study in 716 patients over 8 years. BMC Cardiovasc Disord. 2008;8:27.

32. Anand DV, Lim E, Hopkins D, Corder R, Shaw LJ, Sharp P, et al. Risk stratification in uncomplicated type 2 diabetes: prospective evaluation of the combined use of coronary artery calcium imaging and selective myocardial perfusion scintigraphy. Eur Heart J. 2006;27(6):713-21.

33. Kakuta K, Dohi K, Yamada T, Yamanaka T, Kawamura M, Nakamori S, et al. Detection of coronary artery disease using coronary flow velocity reserve by transthoracic Doppler echocardiography versus multidetector computed tomography coronary angiography: influence of calcium score. J Am Soc Echocardiogr. 2014;27(7):775-85.

34. Kawata T, Daimon M, Hasegawa R, Toyoda T, Sekine T, Himi T, et al. Prognostic value of coronary flow reserve assessed by transthoracic Doppler echocardiography on long-term outcome in asymptomatic patients with type 2 diabetes without overt coronary artery disease. Cardiovasc Diabetol. 2013;12:121.

35. Schachinger V, Britten MB, Zeiher AM. Prognostic impact of coronary vasodilator dysfunction on adverse long-term outcome of coronary heart disease. Circulation. 2000;101(16):1899-906.

36. Galderisi M, Rigo F, Gherardi S, Cortigiani L, Santoro C, Sicari R, et al. The impact of aging and atherosclerotic risk factors on transthoracic coronary flow reserve in subjects with normal coronary angiography. Cardiovasc Ultrasound. 2012;10:20. 